
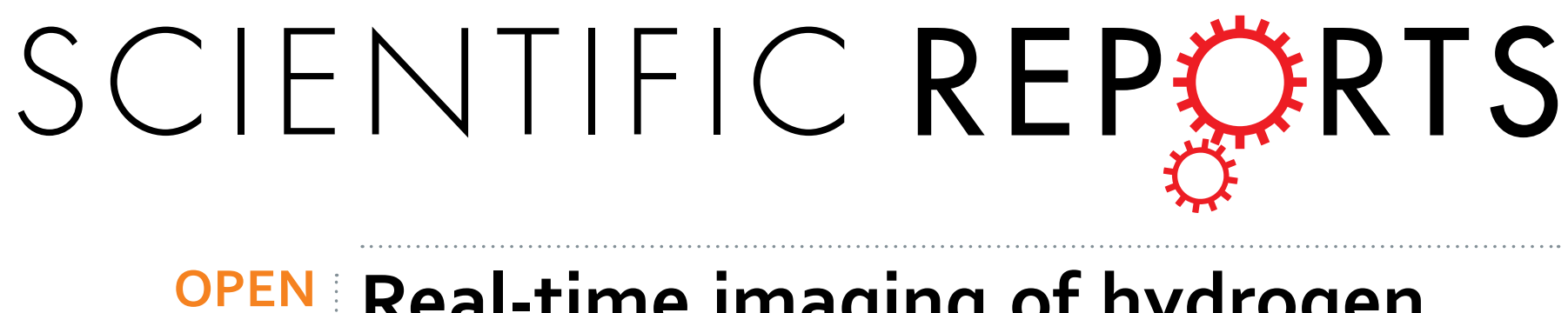

Real-time imaging of hydrogen peroxide dynamics in vegetative and pathogenic hyphae of

Received: 29 June 2015

Accepted: 15 September 2015

Published: 08 October 2015

\section{Fusarium graminearum}

Michael Mentges \& Jörg Bormann

Balanced dynamics of reactive oxygen species in the phytopathogenic fungus Fusarium graminearum play key roles for development and infection. To monitor those dynamics, ratiometric analysis using the novel hydrogen peroxide $\left(\mathrm{H}_{2} \mathrm{O}_{2}\right)$ sensitive fluorescent indicator protein HyPer-2 was established for the first time in phytopathogenic fungi. $\mathrm{H}_{2} \mathrm{O}_{2}$ changes the excitation spectrum of HyPer-2 with an excitation maximum at $405 \mathrm{~nm}$ for the reduced and $488 \mathrm{~nm}$ for the oxidized state, facilitating ratiometric readouts with maximum emission at $516 \mathrm{~nm}$. HyPer-2 analyses were performed using a microtiter fluorometer and confocal laser scanning microscopy (CLSM). Addition of external $\mathrm{H}_{2} \mathrm{O}_{2}$ to mycelia caused a steep and transient increase in fluorescence excited at $488 \mathrm{~nm}$. This can be reversed by the addition of the reducing agent dithiothreitol. HyPer-2 in F. graminearum is highly sensitive and specific to $\mathrm{H}_{2} \mathrm{O}_{2}$ even in tiny amounts. Hyperosmotic treatment elicited a transient internal $\mathrm{H}_{2} \mathrm{O}_{2}$ burst. Hence, HyPer-2 is suitable to monitor the intracellular redox balance. Using CLSM, developmental processes like nuclear division, tip growth, septation, and infection structure development were analyzed. The latter two processes imply marked accumulations of intracellular $\mathrm{H}_{2} \mathrm{O}_{2}$. Taken together, HyPer-2 is a valuable and reliable tool for the analysis of environmental conditions, cellular development, and pathogenicity.

Fusarium graminearum (teleomorph Gibberella zeae) is a necrotrophic, filamentous ascomycete. It infects all cereals and is one of the most important phytopathogens worldwide. Fusarium Head Blight (FHB) of wheat (Triticum aestivum) and barley (Hordeum vulgare) and ear rot of maize (Zea mays), caused by F. graminearum, are of special economic importance in terms of crop losses and accumulation of mycotoxins (reviewed in Sutton, 1982 ${ }^{1}$; Goswami and Kistler, 2004²). Fusarium graminearum forms specialized infection structures, called infection cushions, in order to penetrate the surface of wheat floral leafs $^{3}$. Their formation is important for colonization of the host as a F. graminearum adenylyl cyclase deletion mutant-defective in infection cushion development-fails to penetrate wheat epidermal cells ${ }^{4}$. Inside infection cushions, biosynthesis of trichothecenes takes place ${ }^{3}$. Penetration of the plant surface is accompanied by an unspecific plant response leading to necrosis directly underneath an infection cushion. Infection analysis using a trichothecene-deficient strain revealed that trichothecenes are neither necessary for penetration nor responsible for the formation of necrotic lesions in the plant ${ }^{3}$. Plant necrosis is often related to the production of reactive oxygen species (ROS) ${ }^{5}$. Reactive oxygen species are obvious by-products of aerobic life. They act in a harmful way on membranes, cell walls, proteins, nucleic acids and many other cellular components but, furthermore, also play a certain role in signal transduction. As a matter of first-line defense to invading pathogens, ROS are often produced and secreted by plants (reviewed in Glazebrook, 20056; Heller and Tudzynski, 20117). This immediate and

University of Hamburg, Biocenter Klein Flottbek, Department of Molecular Phytopathology and Genetics, Ohnhorststr. 18, D-22609 Hamburg, Germany. Correspondence and requests for materials should be addressed to J.B. (email: joerg.bormann@uni-hamburg.de) 


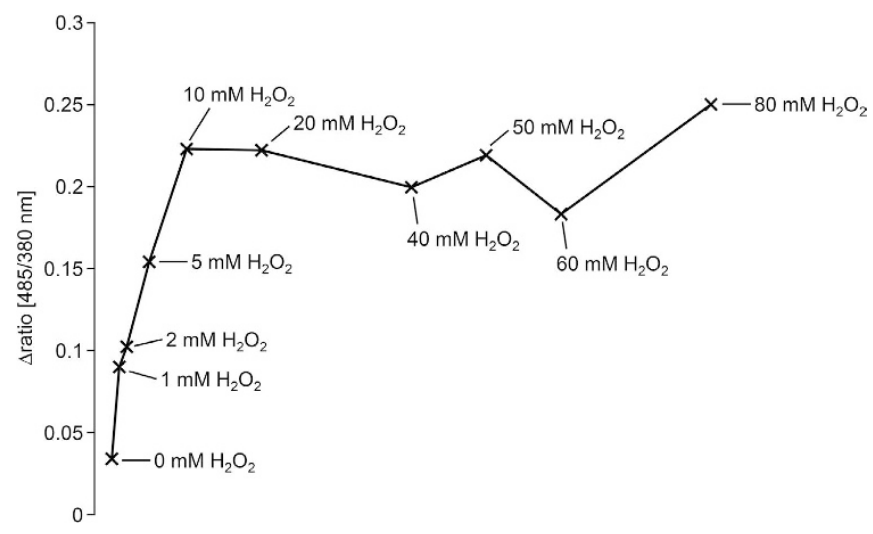

Figure 1. HyPer sensitivity assay. Assay for the relative changes in the ratio $[485 / 380 \mathrm{~nm}]$ in response to increasing $\mathrm{H}_{2} \mathrm{O}_{2}$ concentrations. Mycelia were raised in a 96-well plate filled with $100 \mu \mathrm{l}$ minimal agar medium and analyzed in a fluorometer. Prior to $\mathrm{H}_{2} \mathrm{O}_{2}$ injection $100 \mu \mathrm{l} \mathrm{H} \mathrm{H}_{2} \mathrm{O}$ were injected in each well.

unspecific response, called the oxidative burst, in turn, favors the infection of necrotrophic fungi, since they feed from dead plant material. Fusarium graminearum is widely recognized as a necrotrophic pathogen. However, controversy is ongoing whether or not there might be a brief biotrophic phase early in infection (reviewed in Kazan et al. $2012^{8}$ ). Given this ambiguity it is, to date, not unequivocally known whether or not F. graminearum faces an oxidative burst from the plant during penetration. Balanced production, secretion, and decomposition of ROS are part of the attack strategy of plant pathogenic fungi ${ }^{5,7,9}$. Interferences in the ROS-balance alter the pathogenic potential of $F$. graminearum. Disruption of FgOS-2, the HOG1-homologue of F. graminearum, leads to abnormal catalase gene expression and inappropriate accumulation of ROS. Ultimately, this causes malformed infection cushions (J. Bormann and W. Schäfer, unpublished results) and a drastic reduction in virulence on wheat ${ }^{10}$.

In order to elucidate the ROS-status in fungal mycelia, we established a novel fluorescence bioassay using the genetically encoded fluorescent indicator called $\mathrm{HyPer}^{11}$. Using bio-imaging techniques we analyze ROS-dynamics in mycelia of F. graminearum in vitro and in the early infection stages on wheat. HyPer consists of a circularly permuted yellow fluorescent protein (cpYFP) inserted into the regulatory domain (RD) of the prokaryotic $\mathrm{H}_{2} \mathrm{O}_{2}$-sensing protein, OxyR. Studies from HeLa cells prove a high specificity of this indictor for $\mathrm{H}_{2} \mathrm{O}_{2}{ }^{11,12}$ due to a hydrophobic pocket within OxyR that prevents the attack by charged oxidants such as the superoxide anion radical but allows the penetration of amphiphilic $\mathrm{H}_{2} \mathrm{O}_{2}{ }^{13}$. Upon oxidation, the formation of a disulfide bond mediates a conformational change inside OxyR-RD that is passed on to cpYFP. Oxidation of HyPer, thereby, increases fluorescence of cpYFP excited at $488 \mathrm{~nm}$ and decreases fluorescence excited at $405 \mathrm{~nm}$, respectively. Maximal fluorescence emission is recorded at $516 \mathrm{~nm}$. The sensory functions of HyPer are affected by the ambient $\mathrm{pH}$. To circumvent false readouts and conclusions, $\mathrm{H}_{2} \mathrm{O}_{2}$ insensitive variant of HyPer, called SypHer, was introduced ${ }^{14}$. A point mutation in one of the two $\mathrm{H}_{2} \mathrm{O}_{2}$-sensing cysteine residues of the OxyR-RD domain of Hyper renders the sensor unresponsive to $\mathrm{H}_{2} \mathrm{O}_{2}$, while preserving its $\mathrm{pH}$ sensitivity.

This is the first report on HyPer-fluorescence assays in a phytopathogenic fungus. Its expression in hyphae of $\mathrm{F}$. graminearum provides insights in $\mathrm{H}_{2} \mathrm{O}_{2}$-dynamics inside mycelia of this destructive pathogen.

\section{Results}

HyPer-fluorescence responds specifically to varying amounts of external $\mathrm{H}_{2} \mathrm{O}_{2}$. Fusarium graminearum HyPer and SypHer mutants generated by protoplast transformation of the wild type PH1 were phenotypically characterized regarding vegetative growth, virulence, stress tolerance and fluorescence intensity. Three mutants with strong HyPer (herein referred to as PH1-HyPer mutants) and SypHer (herein referred to as PH1-SypHer mutants) fluorescence in the cytosol, respectively, were selected. Those mutants were similar to wild type regarding growth habit and sensitivity towards oxidative stress (Figure S1). To test responsiveness and specificity of HyPer-2, a microtiter plate assay using a fluorometer was established. An injector attached to the fluorometer facilitates injection of oxidizing and reducing agents, while, simultaneously, measuring fluorescence of mycelia grown on solid minimal medium (MM). A typical measurement cycle comprises the following steps: 1 . measurement of ground-state fluorescence (in a range from $508 \mathrm{~nm}$ to $548 \mathrm{~nm}$ ) after excitation at $380 \mathrm{~nm}$ and $485 \mathrm{~nm}$, 2. fluorescence after injection of a test substance (e.g. $\mathrm{H}_{2} \mathrm{O}_{2}$ ), 3. fluorescence after injection of a second test substance (e.g. dithiothreitol, DTT), 4. if applicable, fluorescence after an additional injection (e.g. $\mathrm{H}_{2} \mathrm{O}_{2}$ ). Afterwards, the ratio of fluorescence recorded after excitation at $485 \mathrm{~nm}$ and $380 \mathrm{~nm}$ (herein after referred to as ratio [485/380 nm]) was calculated. Initially, we tested the sensitivity of HyPer-2 towards $\mathrm{H}_{2} \mathrm{O}_{2}$ in hyphae of F. graminearum. For this, increasing amounts of $\mathrm{H}_{2} \mathrm{O}_{2}(0,1,2,5,10,20,40,60,80 \mathrm{mM}$ (here and from hereon, concentrations are given as final concentrations in the well)) and DTT $(0,5,10,20,40,50 \mathrm{mM})$ 


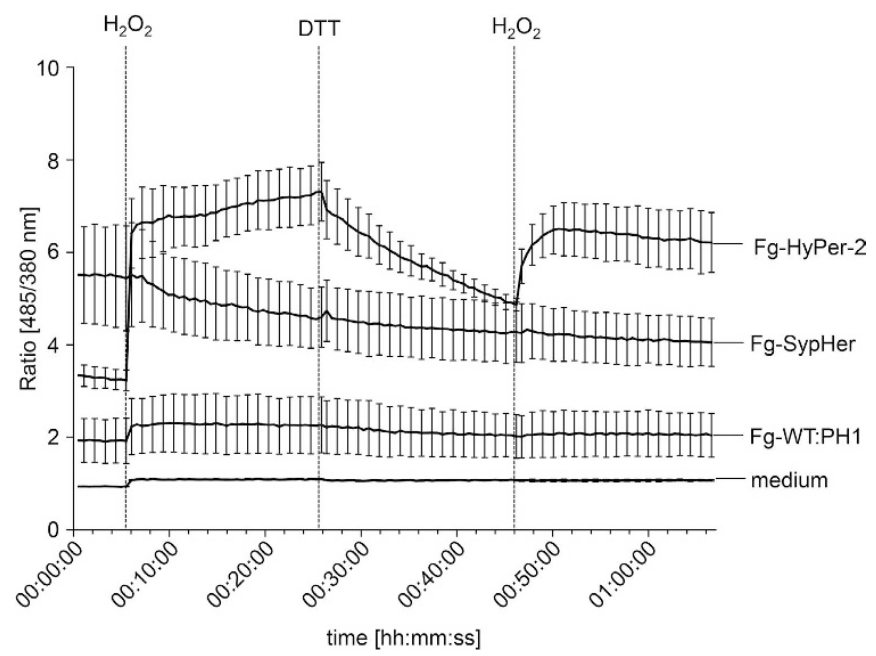

Figure 2. Ratiometric time course assay of the fungal response to external $\mathrm{H}_{2} \mathrm{O}_{2}$ and dithiothreitol (DTT). Timing of $\mathrm{H}_{2} \mathrm{O}_{2}(50 \mathrm{mM})$ and DTT $(50 \mathrm{mM})$ induced ratio [485/380 nm] change in hyphae expressing HyPer-2, SypHer compared to the wild type and media control. Mycelia were raised in a 96-well plate filled with $100 \mu \mathrm{l}$ minimal medium agar and analyzed in a fluorometer. Prior to the first $\mathrm{H}_{2} \mathrm{O}_{2}$ injection $100 \mu \mathrm{l} \mathrm{H}_{2} \mathrm{O}$ were injected in each well. Error bars represent the standard deviation $(\mathrm{n}=24)$.

were added to fungal cultures in the microtiter plate. The ratio [485/380 nm] increased after injection of $\mathrm{H}_{2} \mathrm{O}_{2}$ at concentrations up to $10 \mathrm{mM}$ (Fig. 1). Further increment of $\mathrm{H}_{2} \mathrm{O}_{2}$-concentrations did not evoke a higher ratio $[485 / 380 \mathrm{~nm}]$ indicating a state of maximum oxidation in the presence of $10 \mathrm{mM} \mathrm{H}_{2} \mathrm{O}_{2}$. Viability of fungal hyphae was not compromised after treatment with up to $80 \mathrm{mM} \mathrm{H}_{2} \mathrm{O}_{2}$ (Figure S2). The reduction of HyPer by DTT increased proportionally to the amount of DTT added to the culture. The addition of $10 \mathrm{mM}$ DTT moderately decreased the ratio [485/380 nm]. With higher DTT concentrations, the decrease in ratio $[485 / 380 \mathrm{~nm}]$ got more pronounced (Figure S3).

After sequential addition of $50 \mathrm{mM} \mathrm{H}_{2} \mathrm{O}_{2}$ and $50 \mathrm{mM}$ DTT according to the scheme described above, no significant changes in ratio [485/380 nm] were observed in mycelia of the wild-type and PH1-SypHer and in the media control throughout the entire experimental procedure. This indicates that neither media nor wild type hyphae autofluorescence alters upon $\mathrm{H}_{2} \mathrm{O}_{2}$ or DTT injection and that SypHer, indeed, does not respond to oxidation and reduction. The PH1-HyPer mutants, in contrast, immediately responded to the injection of $\mathrm{H}_{2} \mathrm{O}_{2}$ with an up to 2-fold increase in the ratio [485/380 nm] from $3.2( \pm 0.22)$ to $6.4( \pm 0.75)$ (Fig. 2). As expected ${ }^{11}$, the fluorescence intensity after excitation at $380 \mathrm{~nm}$ (reduced state) moderately decreased, while the fluorescence emitted upon excitation at $485 \mathrm{~nm}$ (oxidized state) strongly increased (Figure S4). The ratio $[485 / 380 \mathrm{~nm}]$ remained on a high level, presumably due to the fact that the $\mathrm{H}_{2} \mathrm{O}_{2}$ was not washed out from the wells. Addition of DTT decreased the ratio [485/380 nm] 1.5-fold compared to the maximum after oxidation. The second injection of $\mathrm{H}_{2} \mathrm{O}_{2}$ again increased the ratio $[485 / 380 \mathrm{~nm}]$ on levels 1.2 -times higher than the level before the first injection. Taken together, these results prove that HyPer-2 is responsive to oxidizing and reducing agents, and suitable for monitoring redox-fluctuations in F. graminearum.

Environmental stimuli induce oxidative bursts in mycelia of HyPer-expressing hyphae. To test the potential of HyPer- 2 to report changes in the internal $\mathrm{H}_{2} \mathrm{O}_{2}$ level, $\mathrm{NaCl}^{15}$ was tested in the microtiter plate assay. The addition of $\mathrm{NaCl}$ increased the ratio [485/380 nm] of HyPer-2. This increase was proportional to the amount of $\mathrm{NaCl}$ added to the culture. While addition of $0.5 \mathrm{M} \mathrm{NaCl}$ did not change ratio $[485 / 380 \mathrm{~nm}]$, it raised from $3.1( \pm 0.04)$ to $3.6( \pm 0.1)$ and from $3.9( \pm 0.04)$ to $5.5( \pm 0.33)$ after addition of $1 \mathrm{M}$ and $2.5 \mathrm{M} \mathrm{NaCl}$, respectively (Fig. 3). The increase in ratio [485/380 nm] in response to osmotic stress indicates a release of $\mathrm{H}_{2} \mathrm{O}_{2}$ inside the hyphae. This effect is $\mathrm{H}_{2} \mathrm{O}_{2}$-specific, since the addition of equimolar amounts of $\mathrm{NaCl}$ to $\mathrm{PH} 1$-SypHer did not alter the ratio [485/380 nm] (Fig. 3).

Taken together, these results show that HyPer is a highly specific sensor to monitor $\mathrm{H}_{2} \mathrm{O}_{2}$ release and subsequent decomposition upon external stresses in hyphae of F. graminearum.

Life cell imaging for real-time monitoring of redox states in hyphae of $\boldsymbol{F}$. graminearum. To analyze HyPer-2-responses on the cellular level confocal laser scanning microscopy (CLSM) was performed. For this, a microfluidic chamber was developed that allows injection of stress agents to mycelia growing on agar, while, simultaneously, recording fluorescence (see methods section for details; Figure S5). After $24 \mathrm{~h}$ of growth in the flow chamber the conidia were germinated and the assay was started $(\mathrm{n}=8)$. Injection of $\mathrm{H}_{2} \mathrm{O}_{2}$ caused an immediate increase in fluorescence after excitation at $488 \mathrm{~nm}$, indicating a rapid oxidation of HyPer-2. Accordingly, fluorescence excited at $405 \mathrm{~nm}$ decreased (Figure S6). 


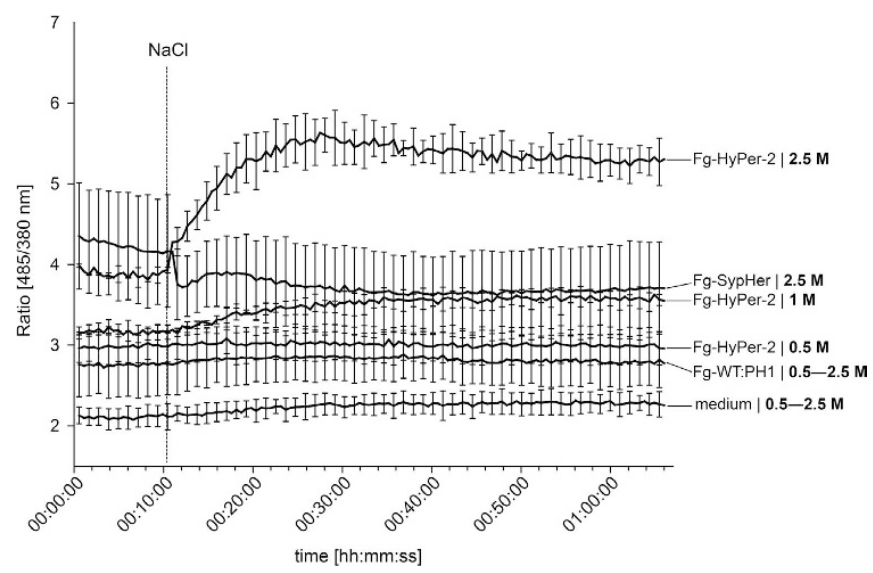

Figure 3. Ratiometric time course assay of the fungal response to osmotic stress. Timing of $\mathrm{NaCl}$ induced ratio [485/380 nm] change in hyphae expressing HyPer-2, SypHer compared to the wild type and media control. Mycelia were raised in a 96-well plate filled with $100 \mu 1$ minimal agar medium and analyzed in a fluorometer. Prior to the $\mathrm{NaCl}$ injection $100 \mu \mathrm{H}_{2} \mathrm{O}$ were injected in each well. Error bars represent the standard deviation $(\mathrm{n}=24)$. Values for the wild type and media control measurements after injection of $\mathrm{NaCl}$ are averaged.

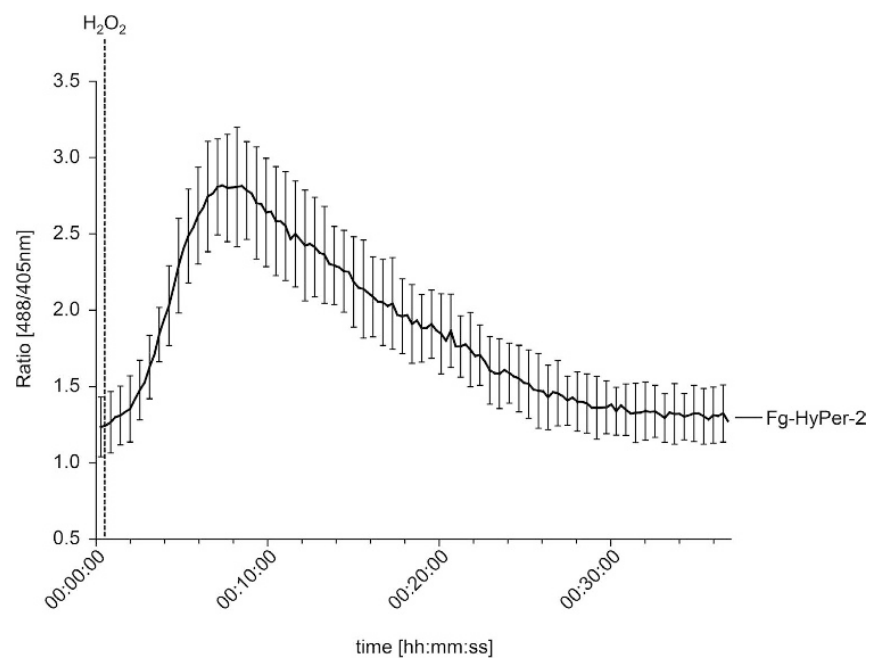

Figure 4. Ratiometric time course assay of the fungal response to external $\mathbf{H}_{2} \mathrm{O}_{2}$. Timing of $\mathrm{H}_{2} \mathrm{O}_{2}$ $(50 \mathrm{mM})$ induced ratio [488/405 nm] change in hyphae expressing HyPer-2. Pixel intensities were measured in three regions of interest marked in a confocal laser scanning time series (see video S1). Error bars represent the standard deviation $(\mathrm{n}=3)$.

The ratio between both channels (ratio [488/405 nm]) increased from approximately 1 to a maximum of 2.8 ( \pm 0.35 ) followed by a slow decrease (Fig. 4, see also video S1). Hence, HyPer-2 enables real-time imaging of rapid oxidative processes inside fungal hyphae using fluorescence microscopy.

Using time-lapse microscopy we analyzed redox fluctuations during growth of fungal hyphae. These analyses included tip growth $(n=10)$, septum formation $(n=6)$, and nuclear divisions $(n=10)$. For the latter we took advantage of a PH1-HyPer-2 mutant expressing a copy of the histon-1 gene tagged with the fluorophore mCherry (A.L. Martínez-Rocha, unpublished results). This facilitates imaging of nuclear dynamics. Fungal growth was imaged over at least one hour taking pictures every $30 \mathrm{~s}$ (image acquisition for each channel took $10 \mathrm{~s}$ including filter exchange). F. graminearum hyphae were growing with a constant rate of around $1.25 \mu \mathrm{m} \mathrm{min}{ }^{-1}$. No spatially distinct accumulations of $\mathrm{H}_{2} \mathrm{O}_{2}$ were detectable in the growing tip of F. graminearum (Figure S7 and video S2). Hence, establishment of polarity axes in hyphal growth seems not to involve $\mathrm{H}_{2} \mathrm{O}_{2}$. Also nuclear divisions during hyphal growth did not provoke fluctuations in intracellular $\mathrm{H}_{2} \mathrm{O}_{2}$ concentrations (Figure S8 and video S3). Septum formation, in contrast, went along with spatially distinct $\mathrm{H}_{2} \mathrm{O}_{2}$ accumulations (Fig. 5, Figure S9 and video S4). To measure fluorescence intensities, regions of interest of equal size were defined both to the right and the left of 


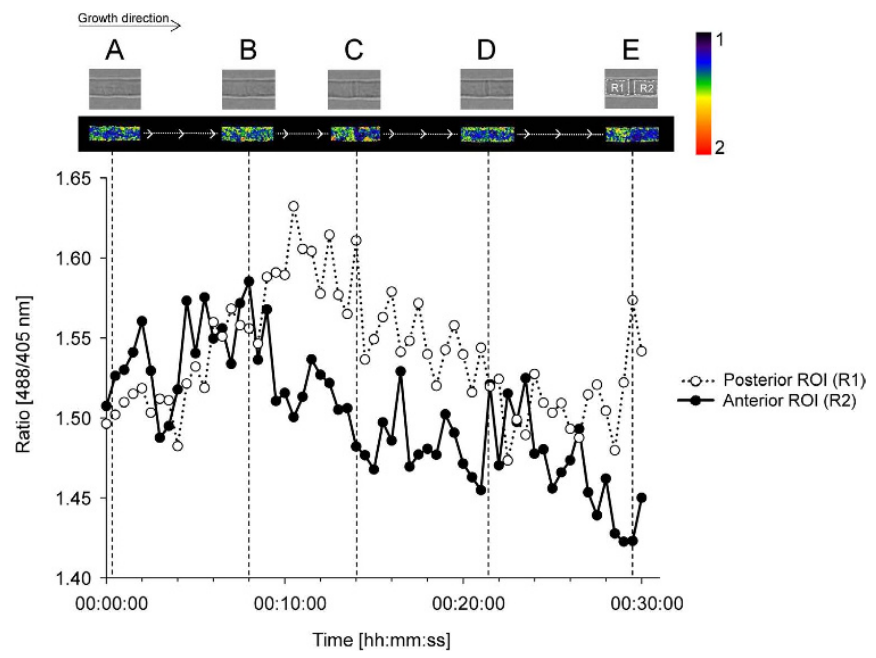

Figure 5. Ratiometric time course assay of septum formation in hypha expressing HyPer-2. Timing of $\mathrm{H}_{2} \mathrm{O}_{2}$ fluctuations during septum formation. Ratio [488/405 nm] calculated from pixel intensities measurements over time in two regions of interest (ROI R1, covering the posterior part and ROI R2, covering the anterior part of the hypha) marked in a confocal laser scanning time series (see also video S4). Annotations (A-E) depict certain developmental stages further explained in the text. Experiments were performed 6 times and gave similar results.

the anticipated location of the septum, representing the anterior and posterior half of the observation area. Prior to septum formation (range between point A and B in Fig. 5), the ratio [488/405 nm] was nearly similar throughout the observation area. After 9 min of observation (shortly after time point B in Fig. 5 and approximately 4 min after initiation of septum formation, indicated as dotted line in Figure S9), the ratio [488/405 $\mathrm{nm}$ ] in the anterior part of the hyphae decreased and indicated lower amounts of $\mathrm{H}_{2} \mathrm{O}_{2}$ compared to posterior part that appeared to be more oxidized. $16 \mathrm{~min}$ after initiation of septum formation, the ratio $[488 / 405 \mathrm{~nm}]$ is again balanced between both parts of the hyphae (time point D in Fig. 5). Interestingly, $23 \mathrm{~min}$ after initiation, the posterior part again got more oxidized than the anterior part (time point E in Fig. 5). Similar experiments using PH1-SypHer did not reveal any significant differences between the ratios $[488 / 405 \mathrm{~nm}]$ in the anterior and posterior part of the hyphae (Figure S10).

These results demonstrate that real-time imaging using HyPer represents a valuable tool to gain a deeper understanding of cell biology in fungi.

HyPer fluorescence assay reveals higher $\mathrm{H}_{2} \mathrm{O}_{2}$-levels in infection cushions of Fusarium graminearum. Infection cushion development can be induced on detached wheat floral organs ${ }^{3}$. Using fluorescent reporter strains, biogenesis of infection cushions emerging from non-infective runner hyphae can be followed over time using CLSM. As expected, the PH1-HyPer mutants developed infection cushions on palea in a wild-type like manner starting 4-5 days post inoculation (dpi). Ratiometric HyPer imaging revealed a greater ratio $[488 / 405 \mathrm{~nm}]$ in infection cushions indicating relatively higher $\mathrm{H}_{2} \mathrm{O}_{2}$-concetrations compared to runner hyphae (Fig. 6A). Figure 6 serves as an example of ratiometric analysis of infection structures and is representative for a series of similar results. For ratio [488/405 nm] calculation regions of interest were defined covering parts of the runner hyphae and the infection cushion (Fig. 6B and C). The ratio [488/405 nm] was approximately 1.3 to 1.5 times the ratio [488/405 nm] of the runner hyphae (Fig. 6D). Therefore, we conclude that the fungus faces localized $\mathrm{H}_{2} \mathrm{O}_{2}$ accumulations specifically in plant-penetrating infection structures. Similar experiments using the PH1-SypHer strains showed no marked differences in the ratio $[488 / 405 \mathrm{~nm}]$ between the runner hyphae and the infection cushions and within either structure (Figure S11).

In conclusion, ratiometric analyses of $\mathrm{H}_{2} \mathrm{O}_{2}$-fluctuations using HyPer-2 offers new insights to regulatory networks and complex interplays between pathogens and their hosts. Furthermore, they are valuable complements to classical genetic approaches for the analysis of cell biology in fungi.

\section{Discussion}

In the present study we analyzed oxidative processes in vegetative and infective hyphae of F. graminearum. To achieve this, we, for the first time in a phytopathogenic fungus, used the ratiometric fluorescent $\mathrm{H}_{2} \mathrm{O}_{2}$-indicator HyPer-2 to monitor fluctuations in $\mathrm{H}_{2} \mathrm{O}_{2}$-level inside hyphae.

HyPer-2 enables monitoring of fluctuations in the intracellular $\mathrm{H}_{2} \mathrm{O}_{2}$-level in response to external stimuli, genetic modifications, and during different developmental stages like nuclear division and septum formation. Ratiometric HyPer-2 imaging uses a genetically encoded sensor protein and, therefore, is 

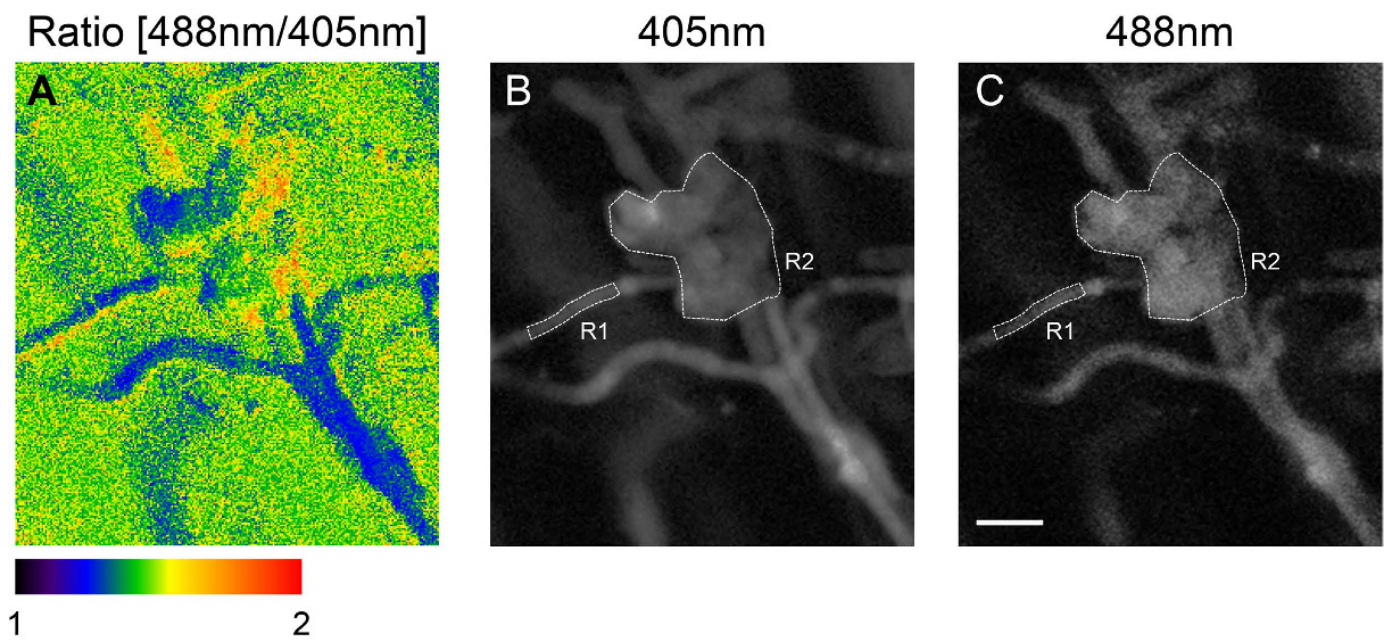

1

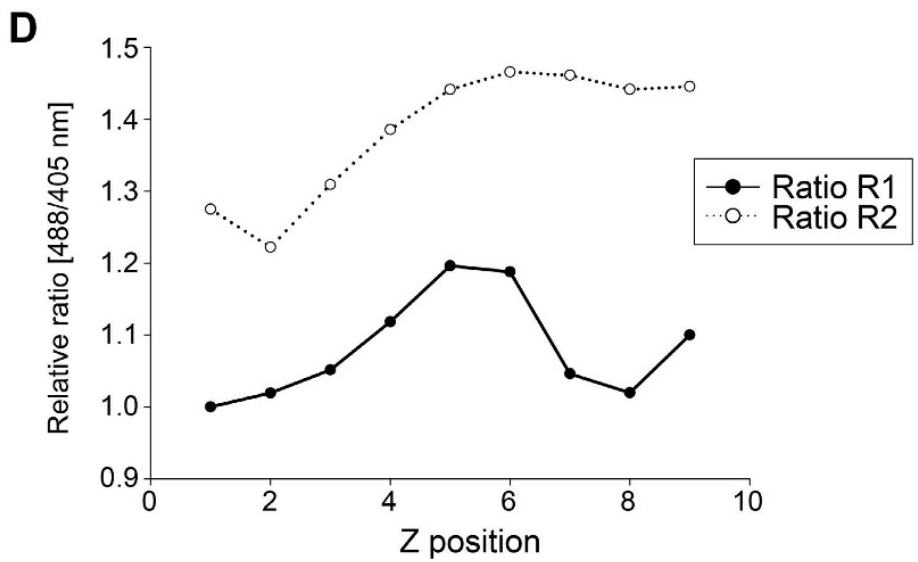

Figure 6. Ratiometric assay of infection cushion formation in hypha expressing HyPer-2. (A-C) Average intensity projections of a tiny infection cushion developed on a wheat palea 6 days post inoculation. (A) Ratio [488/405 nm]. (B) Fluorescence excited at $405 \mathrm{~nm}$. (C) Fluorescence excited at $488 \mathrm{~nm}$. (D) Pixel intensities were measured over nine z-positions in two regions of interest (ROI R1, covering a part of a runner hypha and ROI R2, covering a part of an infection cushion) marked in the confocal laser scanning z-series shown in $(\mathbf{A}, \mathbf{B})$. Analysis of ten different infection structures gave similar results. Scale bar $10 \mu \mathrm{m}$.

equally distributed in the cell or can be targeted to a certain subcellular compartment, e.g. the mitochondria ${ }^{16}$. This makes it advantageous over classical staining methods like $2^{\prime}, 7^{\prime}$-dichlorodihydrofluorescein diacetate $\left(\mathrm{H}_{2} \mathrm{DCFDA}\right.$ ), 3,3'-Diaminobenzidine (DAB), or boronate-based $\mathrm{H}_{2} \mathrm{O}_{2}$ probes (reviewed in Guo et al. $2014^{17}$ ). Technically sophisticated application, insufficient uptake, and inadequate intracellular distribution of stains can, therefore, be avoided. Boronate-based probes are fluorescence turn-on probes with high specificity to $\mathrm{H}_{2} \mathrm{O}_{2}$. They have been widely used for live-cell imaging in non-cell wall systems ${ }^{18}$. Ledoux et al. (2013) ${ }^{19}$ describe the application of this probe in Arabidospsis thaliana. However, enhancement of fluorescence upon oxidation of boronate-based dyes is irreversible and, therefore, limits its applicability for analysis of $\mathrm{H}_{2} \mathrm{O}_{2}$ dynamics. Many other stains (e.g. DAB) do not allow live cell imaging since their application requires harsh conditions like ethanol fixation.

In addition, HyPer-2 has some advantages over other genetically encoded fluorescent indicators. The redox-sensitive GFP2, sometimes coupled to glutaredoxin (GRX), is widely used to analyze redox states in cells indirectly by measuring the thiol redox potential triggered by the electron flow between glutathione and roGFP2 ${ }^{20,21}$. Glutathione is an important and ubiquitous antioxidant. It contains a thiol group that reversibly gets oxidized (forming glutathione disulfide) or reduced by the action of GRX. As depicted before, these indicators do not immediately monitor the redox status of a cell or fluctuation in the $\mathrm{H}_{2} \mathrm{O}_{2}$-concentrations. This limitation can be overcome by in-frame fusion of roGFP2 to the Orp1 peroxidase with high specificity to $\mathrm{H}_{2} \mathrm{O}_{2}{ }^{21}$. However, it cannot be ruled out that constitutive overexpression of GRX or Orp1 coupled to roGFP2 alters the cellular redox status, since both proteins display enzymatic activity in the cell. In HyPer-2, the prokaryotic $\mathrm{H}_{2} \mathrm{O}_{2}$-sensing domain OxyR mediates the conformational change within the cpYFP moiety leading to ratiometric readouts. There are no homologues of this domain in the genome of F. graminearum (e-value: $<1$; data not shown). Hence, HyPer-2 does not interfere with the intracellular ROS-balance and its readout does not rely on enzymatic reactions. Given its biophysical properties it is assumed that HyPer-2 itself, theoretically, can act as an antioxidant ${ }^{13}$. 
However, studies from mammalian systems prove that even strong expression does not interfere with the intracellular ROS-level ${ }^{22,23}$. Previous analyses indicate that the ambient $\mathrm{pH}$ is an issue for HyPer-2 measurements (reviewed in Meyer and Dick, 2010 ${ }^{24}$ ). To rule out any $\mathrm{pH}$-effect in our measurement, we constitutively expressed a $\mathrm{H}_{2} \mathrm{O}_{2}$-insentive HyPer-variant (called SypHer) ${ }^{14}$ in mycelia of F. graminearum and conducted several control experiments. In all assays the SypHer ratio did not significantly change indicating that the intracellular $\mathrm{pH}$ does not interfere with the measurements. Studies from Neurospora crassa demonstrate that the intracellular $\mathrm{pH}$ is tightly controlled and rapidly readjusted after perturbation, e.g. by application of acidic substances ${ }^{25}$.

Reactive oxygen species are unavoidable by-products of a plethora of biochemical processes in the presence of oxygen. Their production and decomposition is usually well balanced and interference with this balance often causes physiological disorders and disease (reviewed i.e. in Taverne et al. 201326; Poljsak et al. 2013 $3^{27}$ ). This insinuates a Janus-faced nature of ROS in cellular processes: opposing a deleterious impact of ROS on lipids, nucleic acids, and proteins they possess profitable function in cell signaling cascades. Localized production of ROS has recently been implicated in the determination of polarity axes and, therefore, in the regulation of directed growth ${ }^{28,29}$. Our results demonstrate the absence of a tip-high $\mathrm{H}_{2} \mathrm{O}_{2}$ accumulation in growing hyphae of $F$. graminearum (Figure S7 and video S2). This suggests that rather superoxide anions $\left(\mathrm{O}_{2}^{-}\right)$accumulate in the hyphal tips. Supportive for this notion is that disruption of $\mathrm{O}_{2}^{-}$generation by functional inactivation of nicotinamide adenine dinucleotide phosphate (NADPH) oxidase (NOX) complex enzymes often cause abnormal apical growth and faulty determinations of polarity axis in fungi ${ }^{30}$.

In the field of phytopathology well balanced ROS-equilibria are also mandatory for proper host-pathogen interaction. Development and pathogenicity of F. graminearum relies on appropriate equilibration of ROS. The deletion of the stress-activated mitogen-acivated protein kinase $\mathrm{FgOS}-2$ and its downstream transcription factor Fgatf1 interfere with ROS-decomposition processes. In consequence, these mutants are defective in maintaining the ROS equilibrium and are, therefore, strongly reduced in virulence $^{10,31}$. More aggressive pathogens like Botrytis cinerea and Leptosphaeria maculans, in contrast, benefit from oxidative bursts derived from themselves and the host ${ }^{32,33}$. Given this diversity in regard to the importance of ROS in host pathogen interaction, we choose to investigate the actual redox state inside pathogenic hyphae.

It is described that F. graminearum preferentially enters wheat floral tissues through natural openings of the plant like stomata ${ }^{34,35}$. More recent findings, however, contradict this notion and substantiate the assumption that specialized and morphological distinct infection structures facilitate immediate penetration of the plant surface and that infection through stomata is more likely to occur stochastically ${ }^{3}$. These structures are structurally dissimilar from dome-shaped appressoria i.e. of Magnaporthe oryzae in which high turgor pressure is present ${ }^{36}$. F. graminearum infection structures appear as lobate, branched and frequently septated hyphal mats that, unlike $M$. oryzae appressoria, develop from epiphytically growing runner hyphae several days post inoculation and not from germinating conidia (see also Emmett and Parbery, 1975 37 ; Mendgen et al. $1996^{8}$ ). For ratiometric analysis of redox states in infective hyphae, we took advantage of the clear morphological distinction between runner hyphae (non-infective) and infection cushions. As depicted in Fig. 6, HyPer is more oxidized in the infection cushion, indicating a local accumulation of $\mathrm{H}_{2} \mathrm{O}_{2}$. By use of roGFP-imaging, Heller et al. (2012) ${ }^{38}$ studied appressoria-like structures of $B$. cinerea. The authors also found a distinct oxidation inside these structures. Hence, localized oxidation inside fungal infection structures is a recurrent pattern in different phytopathogenic fungi that might, therefore, facilitate plant infection. This similar $\mathrm{H}_{2} \mathrm{O}_{2}$ accumulation in the infection structures of the necrotroph B. cinerea and F. graminearum, furthermore, indicate that also F. graminearum acts as a necrotoph already in the earliest infection stages. In the biotrophic fungus Claviceps purpurea which does not differentiate any specialized infection structures ${ }^{39}$, deletion of the $\mathrm{O}_{2}{ }^{-}$-generating NOX subunit NoxA leads to a drastic reduction in virulence. However, hyphae are still able to penetrate the plant ${ }^{40}$. In M. oryzae, in contrast, both NoxA and NoxB are mandatory for plant surface penetration ${ }^{28}$.

Recent results from $M$. oryzae indicate a scaffolding function of NOX-complex proteins in order to align septins and F-actin along the point of penetration peg emergence ${ }^{41}$. This involves localized ROS-production in cell-biological differentiation as it was proposed for developmental processes including sexual reproduction, polarized growth and infection structure development ${ }^{28}$ (reviewed in Scott and Eaton, $2008^{42}$ ). Yet, localized ROS-accumulation inside infection cushions of F. graminearum might promote their formation.

Considering that, at least in M. oryzae, synthesis of ROS by the NOX-complexes directly controls F-actin dynamics, it is reasonable to expect local fluctuations in $\mathrm{H}_{2} \mathrm{O}_{2}$ at the site of septum formation, a process that also implies localized recruitment of F-actin ${ }^{43}$. The fact that we also detected spatially distinct $\mathrm{H}_{2} \mathrm{O}_{2}$ accumulations indicates a similar functional connection between ROS-production and F-actin recruitment for septum formation in F. graminearum. However, thus far not much is known about the genetic and biochemical trigger of septum formation and its suppression. Advancements in recent research (reviewed in Harris, 2012 ${ }^{44}$ ) have shown that localized ROS accumulations are involved in the regulation of septum formation but, undoubtedly, this phenomenon requires further investigation.

NBT staining revealed that growth under high salinity stress conditions increases ROS production ${ }^{31}$. Furthermore, mutants lacking the central regulator of oxidative stress response, the b-zip transcription factor AP1, display a relatively higher sensitivity towards salt stress ${ }^{15}$. By use of HyPer-imaging we now 
demonstrate the intracellular production of $\mathrm{H}_{2} \mathrm{O}_{2}$ in the presence of high amounts of salt in the medium (Fig. 3). This is in accordance to the transcriptional upregulation of potential catalase genes in response to salt stress ${ }^{10}$.

Reactive oxygen species are involved in a plethora of developmental processes in fungi and beyond. Using the fluorescent $\mathrm{H}_{2} \mathrm{O}_{2}$ reporter $\mathrm{HyPer}$ we, for the first time in a phytopathogenic fungus, introduce a highly specific and reliable tool to analyze $\mathrm{H}_{2} \mathrm{O}_{2}$ fluctuations in living cells. Application of HyPer imaging will assist analysis of stress responses and developmental processes in fungi.

\section{Methods}

Generation of plasmids. Standard recombinant DNA methods were performed according to Ausubel et al. (2002) ${ }^{45}$ and Green and Sambrook (2012) et al. (1989) ${ }^{46}$. For stable expression in mycelia of F. graminearum, the entire open reading frame (ORF) of HyPer-2 and SypHer was amplified by PCR using the Q5 proof reading polymerase (New England Biolabs, Frankfurt a. M., Germany) from plasmid pC1-Hyper- $2^{12}$, using primers A1 and A2 (Table S1) and cloned in frame with the promoter of the glyceraldehyd-3-phosphate dehydrogenase from Aspergillus nidulans ( $g$ pdA-promoter) using Bam $\mathrm{HI}$ restriction sites introduced to the primers. Both plasmids (named p7-HyPer-2 and p7-SypHer) were used to transform the F. graminearum wild type PH1.

Generation of mutants. Protoplast transformation of F. graminearum was performed as described previously $^{31}$. Briefly, mycelia were grown overnight in $100 \mathrm{ml}$ YEPG $(0.3 \%$ yeast extract, $1 \%$ bacto peptone, $2 \% \mathrm{D}$-glucose), harvested by filtering, resuspended in $20 \mathrm{ml}$ of enzyme solution containing driselase and lysing enzymes (Life Technologies, Darmstadt, Germany; 2.5\%:0.5\% in $0.6 \mathrm{M} \mathrm{KCl}$ ) and incubated for $2-3 \mathrm{~h}$ at $30^{\circ} \mathrm{C}$. Undigested hyphal material was removed from the protoplast suspension by filtration. The protoplasts were pelleted by centrifugation at $6700 \times \mathrm{g}$, washed once with $10 \mathrm{ml}$ STC $(20 \%$ sucrose, $10 \mathrm{mM}$ Tris- $\mathrm{HCl}, \mathrm{pH} 8.0,50 \mathrm{mM} \mathrm{CaCl}_{2}$ ), centrifuged again, then resuspended and adjusted in STC at $1-6 \times 10^{8}$ protoplasts per ml. For transformation, $200 \mathrm{ml}$ of the protoplast suspension was mixed with approximately $6 \mu \mathrm{g}$ of DNA and incubated at room temperature for $20 \mathrm{~min}$. Subsequently, $1 \mathrm{ml} \mathrm{PEG} \mathrm{(40 \%}$ polyethylene glycol 4000, 60\% STC) was added and again incubated at room temperature for $20 \mathrm{~min}$. The protoplast suspension was added to $50 \mathrm{ml}$ TB3 agar ( $100 \mathrm{~g}$ sucrose, $0.3 \%$ yeast extract, $0.3 \%$ casamino acids, $1.5 \%$ agar) and poured into five $92 \mathrm{~mm}$ petri dishes. After $24 \mathrm{~h}$, an overlay of TB3 agar (1.5\%) containing $200 \mu \mathrm{g} \mathrm{ml}^{-1}$ hygromycin B was added to the plates. Putative transformants were obtained after $2 \mathrm{dpi}$ at $28^{\circ} \mathrm{C}$. They were transferred to fresh plates of complete medium (CM; $1 \mathrm{lCM}$ contained $10 \mathrm{ml}$ of solution $\mathrm{A}\left(100 \mathrm{gl}^{-1} \mathrm{Ca}\left(\mathrm{NO}_{3}\right)_{2} \times 4 \mathrm{H}_{2} \mathrm{O}\right) ; 10 \mathrm{ml}$ of solution $\mathrm{B}\left(20 \mathrm{gl}^{-1} \mathrm{KH}_{2} \mathrm{PO}_{4} ; 25 \mathrm{gl}^{-1} \mathrm{MgSO}_{4} \times 7 \mathrm{H}_{2} \mathrm{O}\right.$; $10 \mathrm{~g} \mathrm{l}^{-1} \mathrm{NaCl}$, sterilized by filtration); $10 \mathrm{~g}$ glucose; $1 \mathrm{ml}$ of suspension $\mathrm{D}\left(60 \mathrm{gl}^{-1} \mathrm{H}_{3} \mathrm{BO}_{3} ; 390 \mathrm{mgl}^{-1}\right.$ $\mathrm{CuSO}_{4} \times 5 \mathrm{H}_{2} \mathrm{O} ; 13 \mathrm{mgl}^{-1} \quad \mathrm{KI} ; 60 \mathrm{mgl}^{-1} \quad \mathrm{MnSO}_{4} \times \mathrm{H}_{2} \mathrm{O} ; 51 \mathrm{mgl}^{-1} \quad\left(\mathrm{NH}_{4}\right)_{6} \mathrm{Mo}_{7} \mathrm{O}_{24} \times 4 \mathrm{H}_{2} \mathrm{O} ; 5.48 \mathrm{gl}^{-1}$ $\left.\mathrm{ZnSO}_{4} \times 7 \mathrm{H}_{2} \mathrm{O} ; 932 \mathrm{mgl}^{-1} \mathrm{FeCl}_{3} \times 6 \mathrm{H}_{2} \mathrm{O}\right), 1 \mathrm{~g}$ yeast extract; $0.5 \mathrm{~g}$ enzymatically hydrolysed casein; $0.5 \mathrm{~g}$ acid-hydrolysed casein) supplemented with $250 \mu \mathrm{g} \mathrm{ml}^{-1}$ hygromycin B. Putative transformants with random integration of the construct were evaluated by fluorescence microscopy (data not shown). Those mutants with high expression of HyPer-2 and SypHer were used for further experiments.

Fluorescence assays. Ratiometric analysis of vegetative hyphae was conducted in two ways: 1 . Fluorometric measurements using multi-well plates and a fluorometer equipped with an injector (Biotek Synergy HT, Biotek, Bad Friedrichshall, Germany), and 2. confocal laser scanning microscopy (Zeiss Axio Imager Z2 with LSM 780 module, Zeiss, Oberkochen, Germany) using objective slides covered with CM-agar and equipped with a custom made injection system (Figure S5).

For fluorometer analysis, each well of black 96-well plates was filled with $100 \mu \mathrm{l} \mathrm{MM-agar} \mathrm{(1.5 \% )}$ (MM; like CM, but without yeast extract and casein). After hardening, media were inoculated with 200 conidia of the wild type and mutant strains expressing HyPer-2 and SypHer, respectively. Fluorescence was exited at $385 / 10 \mathrm{~nm}$ and $485 / 10 \mathrm{~nm}$ and fluorescence emission was monitored at a range from $508 \mathrm{~nm}$ to $548 \mathrm{~nm}$. In a typical experiment, fluorescence was recorded for approximately $5 \mathrm{~min}$ prior to injection of any agent in a given concentration. Each measure cycle after injection was approximately $20 \mathrm{~min}$. Concentrations depicted in the Figure legends and the main text are given as final concentrations after dilution with medium or water present in the wells.

For fluorescence microscopy of in vitro mycelia, conidia of $\mathrm{PH} 1-\mathrm{HyPer}$ mutants were inoculated on objective slides covered with a thin layer of agar surrounded by a double-sided adhesive frame (Gene Frame, Thermo Scientific, Schwerte, Germany, see Figure S5). 12-14hpi, a second frame was installed on top of the first that contained two openings which served as ports for injection and efflux of agents. A cover slip $(24 \times 40 \mathrm{~mm})$ was placed on top of the second frame. Injection of agents was achieved by a syringe attached to a Heidelberger extension (Fresenius Kabi AG, Bad Homburg, Germany) and an endoneedle for root canal rinsing (Vedefar N.V., Dilbeek, Belgium).

For HyPer and SypHer imaging on inoculated wheat paleas, wheat florets were dissected using a razor blade, placed on water agar (1.5\%) and inoculated with 100 conidia. Starting 7 dpi, paleas were screened for infection cushion development using a MZFLIII fluorescence stereomicroscope (Leica Microsystems, Heerbrugg, Switzerland). Positive samples were transferred to objective slides equipped with a Gene 
Frame and analyzed by CLSM. Regions of interest (ROIs) were defined inside runner hyphae and infection cushions and pixel intensities were measured.

HyPer and SypHer were both excited at $405 \mathrm{~nm}$ using a solid-state laser and at $488 \mathrm{~nm}$ using an argon ion laser. Fluorescence was recorded at a range from $508 \mathrm{~nm}$ to $548 \mathrm{~nm}$. For calculation of ratios, regions of interest (ROIs) were defined in which pixel were counted. For ratiometric analysis, photo-multiplier sensitivity was adjusted in a way that excitation at $405 \mathrm{~nm}$ and $488 \mathrm{~nm}$ led to similar fluorescence intensities in the non-stressed situation, leading to a ratio of approximately 1.

\section{References}

1. Sutton, J. C. Epidemiology of wheat head blight and maize ear rot caused by Fusarium graminearum. Nous 4, 195-209 (1982).

2. Goswami, R. S. \& Kistler, H. C. Heading for disaster: Fusarium graminearum on cereal crops. Mol Plant Pathol 5, 515-525 (2004).

3. Boenisch, M. J. \& Schäfer, W. Fusarium graminearum forms mycotoxin producing infection structures on wheat. BMC Plant Biol 11, 110 (2011).

4. Bormann, J., Boenisch, M. J., Brückner, E., Firat, D. \& Schäfer, W. The adenylyl cyclase plays a regulatory role in the morphogenetic switch from vegetative to pathogenic lifestyle of Fusarium graminearum on wheat. PLoS One 9, e91135 (2014).

5. Barna, B., Fodor, J., Harrach, B. D., Pogany, M. \& Kiraly, Z. The Janus face of reactive oxygen species in resistance and susceptibility of plants to necrotrophic and biotrophic pathogens. Plant physiol biochem 59, 37-43 (2012).

6. Glazebrook, J. Contrasting mechanisms of defense against biotrophic and necrotrophic pathogens. Annu Rev Phytopathol 43, 205-227 (2005).

7. Heller, J. \& Tudzynski, P. Reactive Oxygen Species in Phytopathogenic Fungi: Signaling, Development, and Disease. Annu Rev Phytopathol 49, 369-390 (2011).

8. Kazan, K., Gardiner, D. M. \& Manners, J. M. On the trail of a cereal killer: recent advances in Fusarium graminearum pathogenomics and host resistance. Mol Plant Pathol 13, 399-413 (2012).

9. van Kan, J. A. L. Licensed to kill: the lifestyle of a necrotrophic plant pathogen. Trends in Plant Sci 11, 247-253 (2006).

10. Nguyen, T. V., Schäfer, W. \& Bormann, J. The stress-activated protein kinase FgOS-2 is a key regulator in the life cycle of the cereal pathogen Fusarium graminearum. Mol Plant-Microbe In 25, 1142-1156 (2012).

11. Belousov, V. V. et al. Genetically encoded fluorescent indicator for intracellular hydrogen peroxide. Nat Meth 3, 281-286 (2006).

12. Markvicheva, K. N. et al. A genetically encoded sensor for $\mathrm{H}_{2} \mathrm{O}_{2}$ with expanded dynamic range. Bioorg Med Chem 19, 1079-1084 (2011).

13. Lukyanov, K. A. \& Belousov, V. V. Genetically encoded fluorescent redox sensors. Biochim Biophys Acta 1840, 745-756 (2013.).

14. Poburko, D., Santo-Domingo, J. \& Demaurex, N. Dynamic Regulation of the Mitochondrial Proton Gradient during Cytosolic Calcium Elevations. J Biol Chem 286, 11672-11684 (2011).

15. Montibus, M. et al. The bZIP transcription factor Fgap1 mediates oxidative stress response and trichothecene biosynthesis but not virulence in Fusarium graminearum. PLoS ONE 8, e83377 (2013).

16. Malinouski, M., Zhou, Y., Belousov, V. V., Hatfield, D. L. \& Gladyshev, V. N. Hydrogen peroxide probes directed to different cellular compartments. PLoS One 6, e14564 (2011).

17. Guo, H., Aleyasin, H., Dickinson, B. C., Haskew-Layton, R. E. \& Ratan, R. R. Recent advances in hydrogen peroxide imaging for biological applications. Cell Biosci 4, 64 (2014).

18. Lin, V. S., Dickinson, B. C. \& Chang, C. J. Boronate-Based Fluorescent Probes: Imaging Hydrogen Peroxide in Living Systems. Method enzymol 526, 19-43.

19. Ledoux, Q. et al. Validation of the boronate sensor ContPY1 as a specific probe for fluorescent detection of hydrogen peroxide in plants. Plant Signal Behav 8, e26827.

20. Meyer, A. J. et al. Redox-sensitive GFP in Arabidopsis thaliana is a quantitative biosensor for the redox potential of the cellular glutathione redox buffer. Plant J 52, 973-986 (2007).

21. Gutscher, M. et al. Real-time imaging of the intracellular glutathione redox potential. Nat Methods 5, 553-559 (2008).

22. Love, N. R. et al. Amputation-induced reactive oxygen species are required for successful Xenopus tadpole tail regeneration. Nat Cell Biol 15, 222-228 (2013).

23. Niethammer, P., Grabher, C., Look, A. T. \& Mitchison, T. J. A tissue-scale gradient of hydrogen peroxide mediates rapid wound detection in zebrafish. Nature 459, 996-999 (2009).

24. Meyer, A. J. \& Dick, T. P. Fluorescent protein-based redox probes. Antioxid redox sign 13, 621-650 (2010).

25. Parton, R. M. et al. Pronounced cytoplasmic pH gradients are not required for tip growth in plant and fungal cells. J Cell Sci 110 (Pt 10), 1187-1198 (1997).

26. Taverne, Y. J. H. J., Bogers, A. J. J. C., Duncker, D. J. \& Merkus, D. Reactive oxygen species and the cardiovascular system. Oxidative medicine and cellular longevity, Article ID 862423, doi: 10.1155/2013/862423 (2013).

27. Poljsak, B., Šuput, D. \& Milisav, I. Achieving the balance between ROS and antioxidants: when to use the synthetic antioxidants. Oxid Med Cell Longev, Article ID 956792, doi: 10.1155/2013/956792 (2013).

28. Egan, M. J., Wang, Z.-Y., Jones, M. A., Smirnoff, N. \& Talbot, N. J. Generation of reactive oxygen species by fungal NADPH oxidases is required for rice blast disease. P Natl Acad Sci USA 104, 11772-11777 (2007).

29. Takemoto, D., Tanaka, A. \& Scott, B. A p67Phox-like regulator is recruited to control hyphal branching in a fungal-grass mutualistic symbiosis. Plant Cell 18, 2807-2821 (2006).

30. Semighini, C. P. \& Harris, S. D. Regulation of apical dominance in Aspergillus nidulans hyphae by reactive oxygen species. Genetics 179, 1919-1932 (2008).

31. Nguyen, T. V., Kröger, C., Bönnighausen, J., Schäfer, W. \& Bormann, J. The ATF/CREB transcription factor Atf1 is essential for full virulence, deoxynivalenol production and stress tolerance in the cereal pathogen Fusarium graminearum. Mol Plant Microbe In 26, 1378-1394 (2013).

32. Tiedemann, A. V. Evidence for a primary role of active oxygen species in induction of host cell death during infection of bean leaves with Botrytis cinerea. Physiol Mol Plant P 50, 151-166 (1997).

33. Li, C., Barker, S. J., Gilchrist, D. G., Lincoln, J. E. \& Cowling, W. A. Leptosphaeria maculans Elicits Apoptosis Coincident with Leaf Lesion Formation and Hyphal Advance in Brassica napus. Mol Plant Microbe In 21, 1143-1153 (2008).

34. Bushnell, W. R., Hazen, B. E. \& Pritsch, C. in Fusarium head blight of wheat and barley. (eds. K. J. Leonard \& W. R. Bushnell) 44-83 (APS press, 2003).

35. Pritsch, C., Muehlbauer, G. J., Bushnell, W. R., Somers, D. A. \& Vance, C. P. Fungal development and induction of defense response genes during early infection of wheat spikes by Fusarium graminearum. Mol Plant Microbe In 13, 159-169 (2000).

36. de Jong, J. C., McCormack, B. J., Smirnoff, N. \& Talbot, N. J. Glycerol generates turgor in rice blast. Nature 389, 244-244 (1997).

37. Proctor, R. H., Hohn, T. M. \& McCormick, S. P. Reduced virulence of Gibberella zeae caused by disruption of a trichothecene toxin biosynthetic gene. Mol Plant Microbe In 8, 593-601 (1995). 
38. Heller, J., Meyer, A. J. \& Tudzynski, P. Redox-sensitive GFP2: use of the genetically encoded biosensor of the redox status in the filamentous fungus Botrytis cinerea. Mol Plant Pathol 13, 935-947 (2012).

39. Bormann, J. \& Tudzynski, P. Deletion of Mid1, a putative stretch-activated calcium channel in Claviceps purpurea, affects vegetative growth, cell wall synthesis and virulence. Microbiology 155, 3922-3933 (2009).

40. Giesbert, S., Schurg, T., Scheele, S. \& Tudzynski, P. The NADPH oxidase Cpnox1 is required for full pathogenicity of the ergot fungus Claviceps purpurea. Mol Plant Pathol 9, 317-327 (2008).

41. Ryder, L. S. et al. NADPH oxidases regulate septin-mediated cytoskeletal remodeling during plant infection by the rice blast fungus. Proc Natl Acad Sci USA 110, 3179-3184 (2013).

42. Scott, B. \& Eaton, C. J. Role of reactive oxygen species in fungal cellular differentiations. Curr Opin Microbiol 11, 488-493 (2008).

43. Berepiki, A., Lichius, A., Shoji, J.-Y., Tilsner, J. \& Read, N. D. F-Actin Dynamics in Neurospora crassa. Eukaryot Cell 9, 547-557 (2010).

44. Harris, S. D. in Morphogenesis and Pathogenicity in Fungi (eds Pérez Martín, J. \& Di Pietro, A.) 1-20 (Springer, 2012).

45. Ausubel, F. M. et al. (eds) Short protocols in molecular biology: a compendium of methods from current protocols in molecular biology $5^{\text {th }}$ edn (Wiley, 2002).

46. Green, M. R. \& Sambrook, J. (eds) Molecular cloning: a laboratory manual $4^{\text {th }}$ edn (Cold Spring Harbor Laboratory, 2012).

\section{Acknowledgements}

We thank Dr. V. V. Belousov for providing the HyPer-2 and SypHer plasmids, B. Hadeler and C. Kröger for technical assistance, and B. Doormann for critical reading of the manuscript. We also thank A. L. Martínez-Rocha for providing the H1-mCherry plasmid.

\section{Author Contributions}

M.M. and J.B. conceived and conducted the experiments and analyzed the results. All authors reviewed the manuscript.

\section{Additional Information}

Supplementary information accompanies this paper at http://www.nature.com/srep

Competing financial interests: The authors declare no competing financial interests.

How to cite this article: Mentges, M. and Bormann, J. Real-time imaging of hydrogen peroxide dynamics in vegetative and pathogenic hyphae of Fusarium graminearum. Sci. Rep. 5, 14980; doi: 10.1038/srep14980 (2015).

(c) (1) This work is licensed under a Creative Commons Attribution 4.0 International License. The images or other third party material in this article are included in the article's Creative Commons license, unless indicated otherwise in the credit line; if the material is not included under the Creative Commons license, users will need to obtain permission from the license holder to reproduce the material. To view a copy of this license, visit http://creativecommons.org/licenses/by/4.0/ 